\title{
Conf- $9105113--4$
}

\section{(B) Lawrence Berkeley Laboratory UNIVERSITY OF CALIFORNIA}

Physics Division

Presented at the Strings and Symmetries 1991,

Stony Brook, NY, May 20-25, 1991, and to

be published in the Proceedings

\section{Induced Gauge Theories and W Gravity}

K. Schoutens, A. Sevrin, and P. van Nieuwenhuizen

November 1991

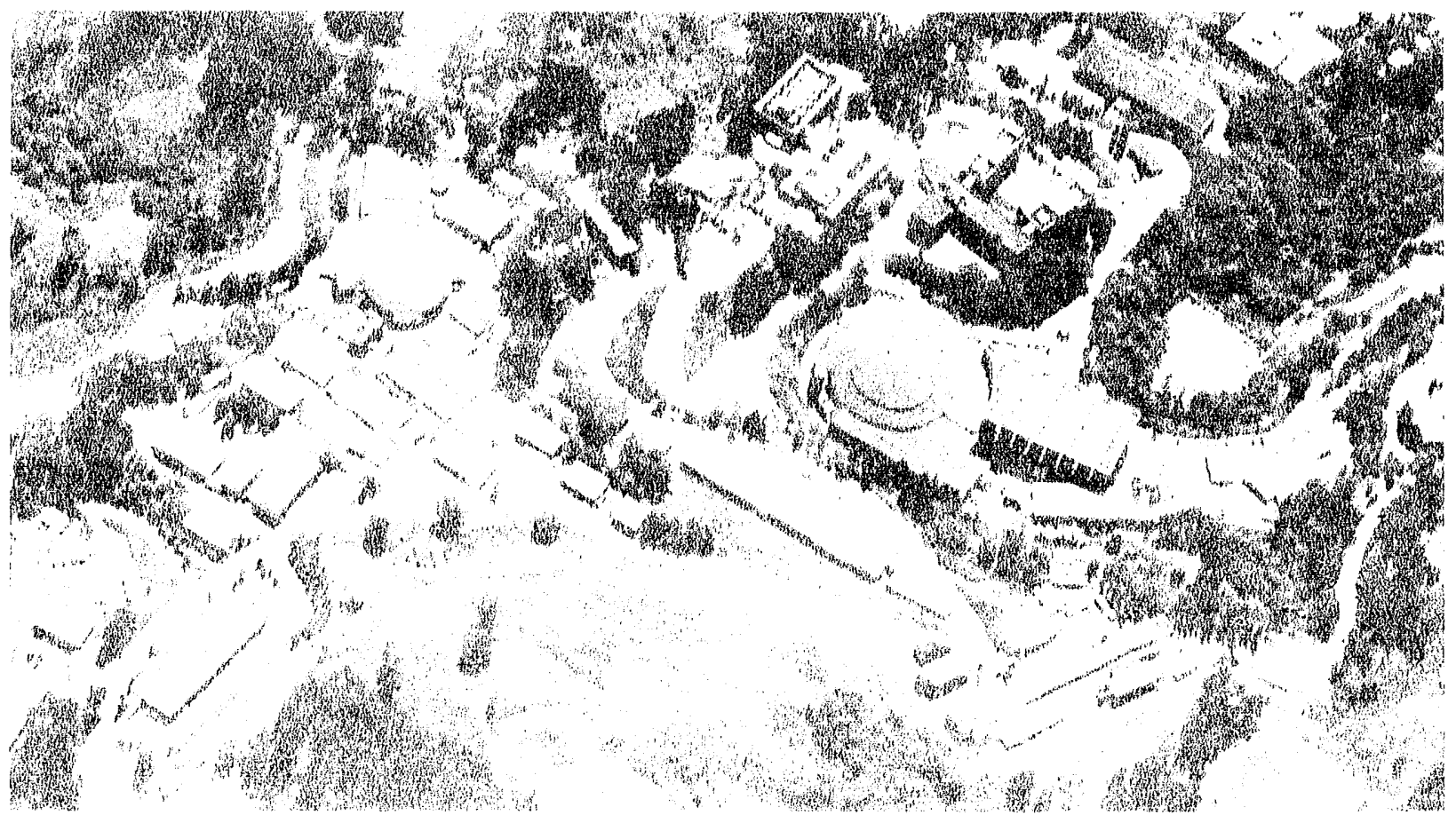

Prepared for the U.S. Department of Energy under Contract Number DE-AC03-76SF00098 


\section{DISCLAIMER}

This document was prepared as an account of work sponsored by the United States Government. Nelther the United States Government nor any agency thereof, nor The Regents of the University of California, nor any of their employees, makes any warranty, express or implied, or assumes any legal liabillty or responsibility for the accuracy, completeness, or usefulness of any information, apparatus, product, or process disclosed, or represents that its use would not infringe privately owned rights. Reference herein to any specific commercial product, process, or service by its trade name, trademark, manufacturer, or otherwise, does not necessarily constitute or imply its endorsement, recommendation, or favoring by the United States Government or any agency thereof, or The Regents of the University of California. The views and opirions of authors expressed herein do not necessarily state or reflect those of the United States Government or any agency thereof or The Regents of the University of California and shall not be used for advertising or product endorsement purposes.

Lawrence Berkeley Laboratory is an equal opportunity employer. 
Nov. 1991

ITP-SB-91-54, CERN-TH.6330/91

LBL-31381, UCB-PTH-91/51

\title{
INDUCED GAUGE THEORIES AND W GRAVITY* ${ }^{* \dagger}$
}

\author{
K. Schoutens ${ }^{1}$, A. Sevrin ${ }^{2}$ and P. van Nieuwerhuizen ${ }^{3,1}$ \\ 1. Institute for Theoretical Physics \\ State University of New York at Stony Brook \\ Stony Brook, NY 11794-3840, U.S.A. \\ 2. Department of Physics \\ University of California at Berkeley \\ and \\ Theoretical Physics group \\ Lawrence berkeley Laboratory \\ Berkeley, CA 94720, U.S.A. \\ 3. Theory division, CERN \\ CH-1211 Geneva 23 \\ Switzerland
}

LBL --31381

DE92 011218

\begin{abstract}
We review some aspects of induced gauge theories in two dimensions. We focus on $\mathrm{W}_{3}$ gravity, paying particular attention to the treatment of the non-linearities inherent to $W$ gravity. We show that the induced action $\Gamma_{\text {ind }}[h, b]$ for chiral $W_{3}$ gravity in the $c \rightarrow \pm \infty$ limit is obtained from the induced action of a gauged $S I(3, R)$ Wess-Zumino-Witten model by imposing constraints on some of the affine currents. Subsequently we investigate the effective action, which is obtained by integrating the induced action over the gauge fields. We show perturbatively that certain subleading terms which appear in the induced action for finite $c$ (and which are related to nonlocal terms in the Ward identities) get cancelled by similar terms due to loop corrections, and we propose an all-order result for the effective action.
\end{abstract}

This report has been reproduced directly from the best available copy.

- Contribution to Strings and Symmetries 1991, Stony Brook, May 1991.

TWork supported in part by grant NSF-91-08054.

tThis work was supported in part by the Director, Office of Energy Research, Office of High Energy and Nuclear Physics, Division of High Energy Physics of the U.S. Department of Energy under Contract DE-AC03-i6SF00098 and in part by the National Science Foundation under grant PHY90-21130. 


\section{Introduction}

Gravity in two dimensions has the exceptional property that it allows for higher spin extensions: the $W$ gravity theories, $[1,2,3]$ (reviews of this field can be found in [4] and especially in [5]).

The basic structure behind these theories are $W$ algebras [6], which are higher spin extensions of the Virasoro algebra. These algebras are not Lie algebras in the usual sense: the commutator of two generators cannot be expressed as a linear combination of generators but contains also composites of the generators,

$$
\left[T_{a}, T_{b}\right]=f_{a b}^{c} T_{c}+V_{a b}^{c d} T_{c} T_{d}+W_{a b}^{c d e} T_{c} T_{d} T_{e}+\cdots
$$

The gauging of quadratically nonlinear algebras was investigated in $[3,7]$. A priori, one would be tempted to treat the composites in the r.h.s. of eq. (1.1) as new generators. This would lead to an infinite dimensional algebra as commuting composites with other generators yields new composites, etc. In $[3,7]$ we showed this complication can be avoided, and that there is a natural way to incorporate the composites in a gauge field theory. Many aspects of the ordinary Lie algebra case carry over to the non-linear case.

In this review we will concentrate on the case of $W_{3}$ gravity, in which the algebra eq. (1.1) has only linear and quadratic terms. A first step in the construction of $W_{3}$ gravity was made by Hull [1], who constructed a classical theory of free scalar fields coupled to $W_{3}$ gravity in the chiral gauge. Subsequently, we extended these results to a light-cone formulation [2], and in [3] we obtained a covariant theory of classical $I V_{3}$ gravity coupled to $n$ scalar fields. A similar program for classical $w_{\infty}$ gravity was carried out in [S].

All of these developments were purely classical. In $W$ gravities, the classical theory (meaning the gauge sector) is in some sense trivial as there are as many gauge field components as local symmetries. In fact, it often occurs in twodimensional conformally invariant theories that a classical theory has as many local gauge invariances as there are gauge field components. Classically, the gauge sector is then pure gauge (up to moduli). However, at the quantum level, some of the symmetries may become anomalous and thereby some gauge degrees of freedom may become propagating.

A typical example for this is provided by a theory of free massless scalar fields coupled to gravity. The gauge multiplet consists of four components: the zweibein. The theory has also four gauge invariances: diffeomorphisms, local Lorentz and Weyl inrariance. Quantizing the theory, one sees that one of these symmetries becomes anomalous. At this point two strategies are available: either one cancels 
the anomaly through a suitable choice of background (which would be 26 scalar fields in this example) so that the over-all coeficient of the integrated anomaly vanishes (the so-called critical approach), or one tries to solve the quantum theory in the presence of propagating gauge field degrees of freedom (the non-critical approach). In the latter case, integrating out the matter fields will result in a quantum action for the gauge fields: the induced action. The action obtained from there by performing a path-integral over the (propagating) gauge fields is called the effective action.

$W_{3}$ gravity is another example of a theory with a non-trivial quantum induced action. The structure of the anomalies in the chiral light-cone gauge has been studied in [9]-[15] (some results in the covariant formulation were obtained in [16]). Contrary to what happens for the gauged WZW model or pure gravity, one finds that the induced action is not proportional to the central charge $c$. Rather, it can be given by a $1 / c$ expansion [10]:

$$
\Gamma_{\text {ind }}[h, b]=\sum_{i \geq 0} c^{1-i} \Gamma^{(i)}[h, b] .
$$

This phenomenon is due to the non-linearities in the $W_{3}$ algebra.

The leading term in eq. (1.2), $\Gamma^{(0)}[h, b]$, is closely related to a gauged $S l(3, \mathbb{R})$ Wess-Zumino-Witen model [14]. The remaining terms in eq. (1.2) do not seem to have a clear group theoretical interpretation. Surprisingly, these subleading terms seem to drop out in the effective theory [15]: it has been found that the first subleading terms in eq. (1.2) are precisely cancelled by certain terms arising from the quantization of the leading term. These remarkable perturbative results led us to the conjecture that the complete effective action (which is the generating functional of 1PI graphs) is, up to multiplicative renormalizations, given by the leading induced action $\Gamma^{(0)}[h, b]$. All-order results for the renormalization constants have been proposed $[15,1 i]$.

As mentioned above, another approach to $W$ gravity, which avoids studying the gauge sector altogether, uses critical theories, i.e., theories which are such that, by a specific choice of the background, all anomalies are made to cancel. By analogy with ordinary gravity, one might expect that this would straightforwardly lead to ' $W$ strings'. However, the construction of $W$ strings has turned out to be much harder than the construction of ordinary bosonic strings. The reason for this is not hard to see. The bosonic string requires a $c=26$ contribution to the central charge from the matter sector for cancellation of the anomaly. The basic Virasoro multiplet consists of one scalar field. Taking 26 copies of this theory and coupling them to gravity indeed yields a viable string theory. In the case of $W_{3}$ strings, the cancellation of all anomalies requires a matter sector which provides an exact 
realization of the $W_{3}$ algebra with central charge $c=100[18,19]$. The basic $W_{3}$ multiplet consists of two scalar fields $(c=2)$. A priori one would expect that taking 50 copies of this theory would save the day. However, the resulting theory is not anymore $W_{3}$ invariant. Its symmetry algebra contains besides the spin 2 and 3 operators also operators of higher spin. One way to obtain $W_{3}$ matter sectors with $c=100$ is through the introduction of background charges in the scalar field theories [1]. This was further analyzed in $[13,20,21,22]$. However, the presence of background charges leads to shifts in the mass formulas for physical string states, so that the existence of massless states in the string-spectrum is in danger (indeed, see [22]). It remains a problem to find a non-trivial $W_{3}$ background with $c=100$ which contains massless spin-2 and possibly higher spin states.

In the next sections we will study three types of induced gauge theories. We will start by reviewing the gauged Wess-Zumino-Witten model, then proceed to study induced gravity, and finally analyze induced and effective $W_{3}$ gravity.

\section{Gauged Wess-Zumino-Witten models}

In this section we review some basic and well-known results on gauged WZW models $[23,24,25,26]$. The gauged WZW model is the prototype induced gauge theory. Many of its characteristic features carry over to induced gravity and $W$ gravity. In fact, we will show that induced gravity and $W$ gravity actions can be obtained from the induced action of a gauged WZW model [14]. This last fact is a manifestation of a deep relation between certain Legendre transforms of gauged WZW models on non-compact groups and gravity theories in two dimensions [2 7 ].

We start with a set of free chiral fermions $\psi=\psi^{a} T_{a}, a=1, \cdots, d$ with action ${ }^{\S}$

$$
S[\psi]=-\frac{1}{2 \pi x} \int d^{2} x \operatorname{tr}\{\psi \bar{\partial} \psi\} \text {. }
$$

The action is invariant under

$$
\delta \psi=[\eta, \psi]
$$

provided

$$
\bar{\partial} \eta=0 \text {. }
$$

\footnotetext{
We normalize such that if $\left[T_{a}, T_{b}\right]=f_{a b}{ }^{c} T_{c}$ then $f_{a c}{ }^{d} f_{b d}{ }^{c}=-\tilde{h} g_{a b}$, where $\tilde{h}$ is the dual Coxeter number. In a representation $R$ we have $\operatorname{tr}\left(T_{a} T_{b}\right)=-x g_{a b}$, where $x$ is the index of the representation ( $x=\bar{h}$ for the adjoint representation). For $S l(n, R)$ one has $\bar{h}=n$ and $x=\frac{1}{2}$ for the vector (defining) representation. Finally, we always work in a two-dimensional Euclidean space. We will use complex coordinates and denote them by $x$ and $\bar{x}$ (or $z$ and $\bar{z}$ ) instead of $x^{+}$ and $x^{-}$.
} 
The Noether currents associated to this symmetry are given by

$$
J=\psi \psi, \quad J_{a}=\frac{1}{2} f_{a b c} \psi^{b} \psi^{c}
$$

and their current algebra is an affine Lie algebra, defined by the following OPE's

$$
J_{a}(x) J_{b}(y)=-\frac{k}{2} g_{a b}(x-y)^{-2}+(x-y)^{-1} f_{a b}^{c} J_{c}(y)+\cdots
$$

In the free fermion example the level is $k=0$ classically (i.e., only single contractions) and $k=\tilde{h}$ quantum mechanically (i.e., also double contractions are made). Introducing a chiral gauge field $A$ allows one to relax the conditions eq. (2.3). Indeed, if we couple the gauge fields in a minimal way:

$$
S[\psi, A]=-\frac{1}{2 \pi x} \int d^{2} x \operatorname{tr}\{\psi \bar{D}[A] \psi\}=-\frac{1}{2 \pi x} \int d^{2} x \operatorname{tr}\{\psi \bar{\partial} \psi+2 J A\}
$$

then the action is invariant under arbitrary variations of the form eq. (2.2) provided the gauge fields transform as

$$
\delta A=\bar{\partial} \eta+[\eta, A]
$$

We now consider the so-called induced action for the gauge fields $A$, to be clenoted by $\Gamma[. A]$, which is the generating functional for current correlation functions:

$$
e^{-\Gamma[A]}=\left\langle\exp -\frac{1}{\pi x} \int d^{2} x \operatorname{tr}\{J(x) A(x)\}\right\rangle \text {. }
$$

In terms of Feynman diagrams it reads

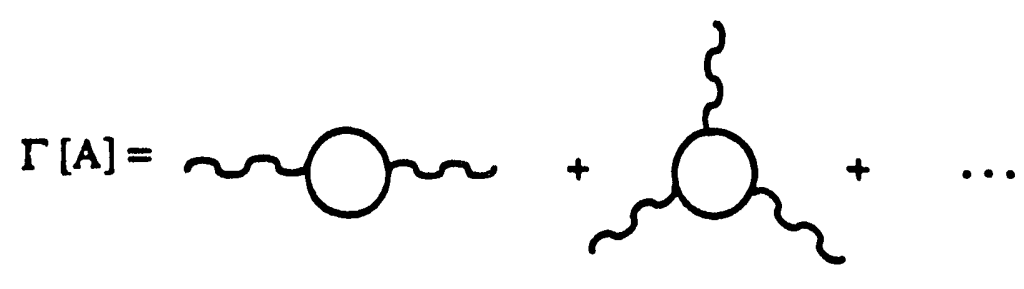


Introducing the notation $\delta_{0} A \equiv \bar{\eta} \eta$ and $\delta_{1} A \equiv[\eta, A]$, one easily shows that for $n \geq 3$

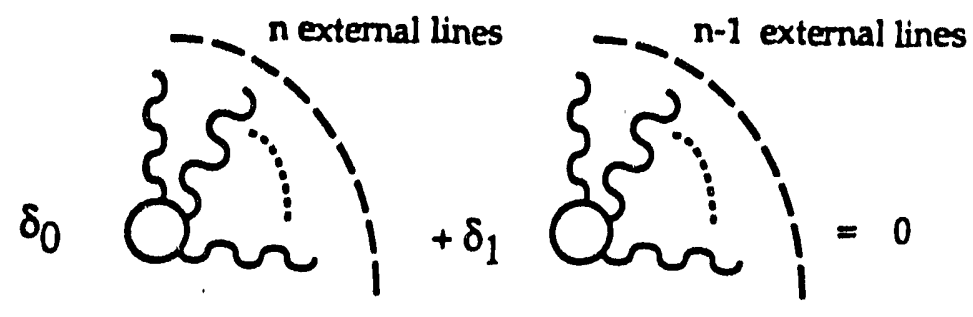

There is thus an anomaly which arises from the lowest order (two-point) diagram. Explicitly computing the variation yields

$$
\delta \Gamma[A]=-\frac{k}{2 \pi x} \int d^{2} x \operatorname{tr}\{\eta \partial A\} .
$$

Defining

$$
u_{a}(x)=-\frac{2 \pi}{k} \frac{\delta \Gamma[A]}{\delta A^{a}(x)}
$$

we deduce from eqs. (2.11) and (2.7) the following Ward identity

$$
\bar{\partial}_{u}-[A, u]=\partial A .
$$

This Ward identity is to be viewed as a functional differential equation for $\Gamma[A]$. It has the following formal solution

$$
\Gamma[A]=\frac{k}{2 \pi x} \int d^{2} x \operatorname{tr}\left\{A \sum_{n \geq 0} \frac{1}{n+2}\left(\left[\frac{1}{\partial} A, \cdot\right]\right)^{n} \frac{\partial}{\partial} A\right\} .
$$

In $[24,25]$ an elegant alternative formulation was developed for $\Gamma[A]$. One observes that eq. (2.13) states that the curvature for the Yang-Mills fields $\{A, u\}$ vanishes. This condition is solved by parametrizing $A$ as $A \equiv \bar{\partial} g g^{-1}$ and $u$ as $u \equiv \partial g g^{-1}$. In this parametrization, eq. (2.7) becomes $\delta g=\eta g$ and eq. (2.11) becomes the equation of motion for $\Gamma[A(g)]$,

$$
\delta \Gamma=-\frac{k}{2 \pi x} \int d^{2} x \operatorname{tr}\left\{\partial\left(\bar{\partial} g g^{-1}\right) \delta g g^{-1}\right\}
$$


which is recognized as the equation of motion for the Wess-Zumino-Witten action

$$
\Gamma[g]=-\frac{k}{4 \pi x} \int d^{2} x \operatorname{tr}\left\{\partial g^{-1} \partial g\right\}-\frac{k}{12 \pi x} \int d^{3} x \epsilon^{\alpha \beta \gamma} \operatorname{tr}\left\{g_{, \alpha} g^{-1} g_{, \beta} g^{-1} g_{, \gamma} g^{-1}\right\}
$$

with $d^{3} x=d x^{3} d x^{+} d x^{-}$and $\epsilon^{3+-}=-1$. Note that the level of the WZW model is given by $-k$.

It is also easy to find the covariant induced action. Indeed,

$$
\Gamma[A, \bar{A}]=\Gamma[A]+\Gamma[\bar{A}]-\frac{k}{2 \pi x} \int d^{2} x \operatorname{tr}\{A(x) \bar{A}(x)\}
$$

is invariant under eq. (2.7) and

$$
\delta \bar{A}=\partial \eta+[\eta, \bar{A}]
$$

Before proceeding we introduce two reference functionals: $S[A]$ and $\bar{S}[t]$. The first one is such that $S\left[A=\delta g g^{-1}\right]$ is the WZW action with $k=1$. The second one is such that $\bar{S}\left[t=\partial g g^{-1}\right]$ is also a $k=1 \mathrm{WZW}$ action, this time with the sign of the Wess-Zumino term reversed. We have the following relation

$$
S[A(t)]+\bar{S}[t]=-\frac{1}{2 \pi x} \int d^{2} x \operatorname{tr}\{A(t) t\}
$$

where $A(t)$ is determined by $t_{a}=2 \pi \frac{\delta}{\delta A^{a}} S[A]$. Similarly,

$$
S[A]+\bar{S}[t(A)]=-\frac{1}{2 \pi x} \int d^{2} x \operatorname{tr}\{A t(A)\}
$$

with $t(A)$ determined through $A^{a}=2 \pi \frac{\delta}{\delta t_{a}} \bar{S}[t]$.

From the free fermion theory we obtain an induced action given by

$$
-\frac{1}{2} \ln \operatorname{det} \bar{D}[A]=-\tilde{h} S[A] \text {. }
$$

Taking an arbitrary matter sector (with current algebra of level $k$ as in eq. (2.5)) leads to an induced action given by

$$
\Gamma[A]=-k S[A]
$$

To quantize also the gauge fields, we now consider the following functional integral

$$
e^{-W[t]}=\int[d A] e^{-\Gamma[A]+\frac{k}{2 \pi x} \int d^{2} x \operatorname{tr}(t A)} \equiv \int[d A] e^{-\Gamma[A, t]}
$$


$W[t]$ is the generating functional of connected diagrams with propagating $A$ fields. A simple Legendre transform of this functional yields the generating functional for 11'I diagrams.

We will approach the computation of the path-integral eq. (2.23) in three different ways: using the saddle point technique, using the fact that the underlying theory is a WZW model and finally by using the KPZ method.

The saddle point method starts by expanding $\Gamma[A, t]$ around the classical configuration of $A$, denoted by $A_{c l}$, which is formally given by

$$
A_{\mathrm{cl}}=(\partial-[t, \cdot])^{-1} \bar{\partial} t
$$

Including first order quantum corrections we have

$$
e^{-W[t]} \simeq e^{-W_{\mathrm{cl}}[t]}\left\{\operatorname{det}\left(\left.\frac{\delta^{2} \Gamma[A, t]}{\delta A^{a} \delta A^{b}}\right|_{A=A_{\mathrm{cl}}}\right)\right\}^{-1 / 2} .
$$

It is clear that the first term $W_{\mathrm{cl}}[t]$ is given in terms of the reference functional

$$
W_{\mathrm{cl}}[t]=k \bar{S}[t] .
$$

Using the Ward identity eq. (2.13), one shows that

$$
\operatorname{det}\left(\left.\frac{\delta^{2} \Gamma[A, t]}{\delta A^{a} \delta A^{b}}\right|_{A=A_{\mathrm{cl}}}\right)=\frac{\operatorname{det} D[t]}{\operatorname{det} \bar{D}\left[A_{\mathrm{cl}}(t)\right]}=e^{2 \tilde{h} \bar{S}[t]-2 \tilde{h} S\left[A_{\mathrm{cl}}(t)\right]} .
$$

Using eqs. (2.19) and (2.25-2.27) we find

$$
W[t]=(k+2 \tilde{h}+\cdots) \bar{S}\left[\left(1-\frac{\tilde{h}}{k}+\cdots\right) t\right] .
$$

For compact groups the level has to be an integer, in that case the first order correction for the level gives the full result. The effective action, which is the generating functional for 1 PI diagrams,

$$
\Gamma_{1 P I}[A]=\min _{\{t\}}\left(W[t]+\frac{k}{2 \pi x} \int d^{2} x \operatorname{tr}(t A)\right),
$$

is now simply given by

$$
\Gamma_{1 P I}[A]=-(k+2 \tilde{h}) S\left[\left(1-\frac{\tilde{h}}{k}+\cdots\right) A\right] .
$$


Comparing with the induced action eq. (2.22), we see that only multiplicative renormalizations have occurred.

Another method relies on the fact that we know that $\Gamma[A]$ is a WZW model and that we know how to quartize a WZW model. Performing the change of variables from $A$ to $g$ yields a Jacobian, which precisely shifts the level from $-k$ to $-k-2 \tilde{h}$. From [28] we know that at the quantum level the effective current $A=\delta g g^{-1}$ gets renormalized to $A=\frac{k+h}{k} \partial g g^{-1}$. This suggests an exact result for $\Gamma_{1 P I}[A]$ :

$$
\Gamma_{1 P I}[A]=-(k+2 \tilde{h}) S\left[\frac{k}{k+\tilde{h}} A\right],
$$

in agreement with eq. (2.30).

Finally, the last method is the KPZ method [29]. There one starts with the fully covariant action, eq. (2.17). One first fixes the gauge by putting $\bar{A}=\overline{\bar{A}}$ where $\overline{\bar{A}}$ is some background configuration. Introducing ghosts and anti-ghosts, the gauge fixed action becomes

$$
\Gamma[A, \hat{\bar{A}}, b, c]=\Gamma[A, \hat{\bar{A}}]+\frac{1}{2 \pi x} \int d^{2} x \operatorname{tr}\left(b \partial c+2 \bar{J}_{\text {ghost }} \hat{\bar{A}}\right) .
$$

In order that the theory does not depend on the gauge choice one has that

$$
\left\langle\frac{\delta \Gamma[A, \hat{\bar{A}}, b, c]}{\delta \hat{\bar{A}}}\right\rangle=0
$$

In particular, the two point function of the currents has to vanish. So the level of the total current which consists of the sum of $\bar{J}_{\text {matter }}, \bar{J}_{\text {ghost }}$ and $A$ has to vanish. The first has level $k$, the second has a level $2 \hat{h}$ which implies that $A$ has level $-k-2 \tilde{h}$. This reasoning thus confirms the overall factor in the result eq. (2.31).

\section{Induced gravity}

Consider a free scalar field $\phi$, coupled to gravity in the chiral gauge (i.e., the only non-trivial component of the metric is $h_{--}$). Its action is given by

$$
S[\phi, h]=\frac{1}{2 \pi} \int d^{2} x(\partial \phi \bar{\partial} \phi+2 h T),
$$

where

$$
T=-\frac{1}{2} \partial \phi \partial \phi
$$


Classically, this action still has a local invariance

$$
\begin{aligned}
\delta \phi & =\epsilon \partial \phi \\
\delta h & =\partial \epsilon+\epsilon \partial h-\partial \epsilon h .
\end{aligned}
$$

We expect this invariance to be broken at quantum level. The induced action $\Gamma[h]$ is defined by

$$
e^{-\Gamma[h]}=\left\langle\exp -\frac{1}{\pi} \int d^{2} x T(x) h(x)\right\rangle,
$$

where the energy-momentum tensor satisfies the OPE

$$
T(x) T(y)=\frac{c}{2}(x-y)^{-4}+2(x-y)^{-2} T(y)+(x-y)^{-1} \partial T(y)+\cdots
$$

In the case of the scalar field one has $c=1$. We will now consider the induced action eq. (3.4) for a general conformal matter system of central charge $c$. In exactly the same way as in the previous section, one shows that there is an $\epsilon$ anomaly, which arises solely from the two-point diagram. It has the form

$$
\delta \Gamma[h]=-\frac{c}{12 \pi} \int d^{2} x \epsilon \partial^{3} h
$$

Defining the effective current

$$
u(x)=\frac{12 \pi}{c} \frac{\delta \Gamma[h]}{\delta h(x)}
$$

we obtain the Ward identity

$$
(\bar{\partial}-2 \partial h-h \partial) u=\partial^{3} h
$$

which is to be viewed as a functional differential equation for $\Gamma[h]$. Though the answer for $\Gamma[h]$ is well-known [30], we will bere derive it again to show a technique which will useful for us later in the case of $W_{3}$ [14].

Consider a gauged $S l(2, \mathrm{R})$ WZW model with level $k$ in the chiral gauge. We choose the basis for the Lie algebra as

$$
T_{0}=\left[\begin{array}{rr}
1 & 0 \\
0 & -1
\end{array}\right], \quad T_{+}=\left[\begin{array}{ll}
0 & 1 \\
0 & 0
\end{array}\right], \quad T_{-}=\left[\begin{array}{ll}
0 & 0 \\
1 & 0
\end{array}\right]
$$


We now reduce this model by imposing constraints on the currents $u$

$$
u=\left[\begin{array}{ll}
0 & u^{+} \\
1 & 0
\end{array}\right]
$$

Using (3.10) and (2.13) we can eliminate $A^{+}$and $A^{0}$ as independent variables, giving

$$
A=\left[\begin{array}{cc}
\frac{1}{2} \partial A^{-} & -\left(\frac{1}{2} \partial^{2} A^{-}-u^{+} A^{-}\right) \\
A^{-} & -\frac{1}{2} \partial A^{-}
\end{array}\right],
$$

and the Ward identities reduce to a single non-trivial equation

$$
\left(\bar{\partial}-2 \partial A^{-}-A^{-} \partial\right) u^{+}=-\frac{1}{2} \partial^{3} A^{-}
$$

Comparing this with eq. (3.8), we see that they are the same if we identify

$$
\begin{aligned}
& h \equiv A^{-} \\
& u \equiv-2 u^{+} .
\end{aligned}
$$

This observation implies that one can obtain $\Gamma[h]$ from $\Gamma[A]$ by a Legendre transformation. Indeed, $u^{+}$is defined by

$$
u^{+}=\frac{\pi}{k} \frac{\delta \Gamma[A]}{\delta A^{-}} \quad \text { at } \quad A^{0}=A^{0}\left(A^{-}\right), \quad A^{+}=A^{+}\left(A^{-}\right)
$$

On the other hand, the object $u(x)$ in pure gravity is obtained by varying an effective action $\Gamma[h]$. Reversing the order in which we differentiate w.r.t. $A^{-}$and impose constraints, we find from the chain rule

$$
u^{+}=\frac{\pi}{k} \frac{\delta}{\delta A^{-}}\left\{\Gamma\left[A^{-}, A^{+}\left(A^{-}\right), A^{0}\left(A^{-}\right)\right]-\frac{k}{\pi} \int d^{2} x u^{+} A^{-}\right\} .
$$

From (3.13) and (3.7) we bave

$$
u^{+}=-\frac{1}{2} u=-\frac{6 \pi}{c} \frac{\delta \Gamma[h]}{\delta h}
$$

Combining eqs. (3.15) and (3.16) yields

$$
\Gamma[h]=-\frac{c}{6 k}\left\{\Gamma\left[A^{-}, A^{+}\left(A^{-}\right), A^{0}\left(A^{-}\right)\right]-\frac{k}{\pi} \int d^{2} x u^{+} A^{-}\right\} .
$$


We can thus conclude that the induced action for gravity in the light-cone gauge is given by a Legendre transform of a constrained WZW model of level $c / 6$. The relation $k=c / 6$ is the leading or classical term in the so-called KPZ-formula, which relates the central charge $c$ of the gravity sector to the $S l(2, \mathrm{R})$ level $k$ $[29,31,32]$.

In [27] we develop a framework in which the constraints eq. (3.10) arise naturally as gauge choices combined with going to a second order formalism (i.e., eliminating auxiliary fields).

A local expression for $\Gamma[h]$ is obtained by using a Gaussian parametrization for $\operatorname{Sl}(2, \mathbf{R})$

$$
g=\left(\begin{array}{ll}
1 & \phi \\
0 & 1
\end{array}\right)\left(\begin{array}{cc}
\lambda^{-1} & 0 \\
0 & \lambda
\end{array}\right)\left(\begin{array}{ll}
1 & 0 \\
f & 1
\end{array}\right)
$$

Solving the constraints in (3.10) yields

$$
\begin{aligned}
\phi & =-\frac{1}{2} \frac{\partial^{2} f}{\partial f} \\
\lambda^{2} & =\frac{1}{\partial f}
\end{aligned}
$$

and

$$
\begin{aligned}
& u=(\partial f)^{-2}\left(\partial^{3} f \partial f-\frac{3}{2} \partial^{2} f \partial^{2} f\right) \\
& h=\frac{\partial f}{\partial f},
\end{aligned}
$$

where we used eq. (3.13) and the fact that $A=\bar{\partial} g g^{-1}$ and $u=\partial g g^{-1}$.

Substituting the Gaussian decomposition into the action in (3.17) one obtains

$$
\begin{aligned}
\Gamma[h] & =\frac{c}{72 \pi} \int d^{3} x \epsilon^{\alpha \beta \gamma}\left[-6 \lambda \partial_{\alpha} \phi \partial_{\beta} f \partial_{\gamma} \lambda\right] \\
& =\frac{c}{24 \pi} \int d^{2} x \frac{\bar{\partial} f}{\partial f} \partial^{2}(\ln \partial f),
\end{aligned}
$$

which is indeed the action for induced gravity in the light-cone gauge.

The transformation rules which give rise to the anomaly can be read off from the Ward identity. However they can also be immediately obtained from the present construction. From $\delta u=\partial \eta+[\eta, u]$ and the constraints, one gets

$$
\eta=\left[\begin{array}{cc}
\frac{1}{2} \partial \eta^{-} & -\left(\frac{1}{2} \partial^{2} \eta^{-}-u^{+} \eta^{-}\right) \\
\eta^{-} & -\frac{1}{2} \partial \eta^{-}
\end{array}\right] .
$$


Eq. (2.7) implies then

$$
\delta h=\bar{\partial} \epsilon+\epsilon \partial h-\partial \epsilon h
$$

(as in eq. (3.3)), where $\epsilon=\eta^{-}$. The stress tensor transforms according to

$$
\delta u=\partial^{3} \epsilon+\epsilon \partial u+2 \partial \epsilon u .
$$

Comparing this analysis to the work in [32], we see that the difference lies in the constraint imposed on $u$. While in the present work we impose the constraints $u^{-}=$constant and $u^{0}=0$, in [32], one imposed $u^{-}=$constant and $\phi=0$. It is interesting to note that while our constraints identify $f$ with the coordinate transformation $y(x, \bar{x})$, the choice of [32] (see also [33]) resulted in the identification of $f$ with the inverse transformation $F$ defined through

$$
F(y(x, \bar{x}), \bar{x})=x .
$$

In the work of [32], the induced action for gravity in terms of the $F$ variable arose completely from the kinetic term of the Wess-Zumino-Witten action, while here it arises from the Wess-Zumino term.

The last step to be performed is the analysis of the effective theory. This was done in $[30,29,31]$ for a fixed (trivial) topology. Again there are various ways to obtain the effective action. The first one is the saddle-point method. One finds that at the saddle point the Ward identity eq. (3.8) implies that when $\partial^{3} h=0$, the three modes of $h$ satisfy an $S l(2, R)$ affine current algebra. This is hardly surprising if one looks back at eq. (3.11). At the saddle point the central charge $c$ is related to the level $k$ of the current algebra as $k=c / 6$. Again a fact which followed from the previous analysis. Through the computation of the Gaussian correction to the saddle point, one finds that, through order $1 / c$, the 1PI (effective) action is related to the induced action by multiplicative renormalizations. On the basis of the KPZ analysis [29], the following all-order result has been proposed [31]

$$
\Gamma_{1 P I}[h]=\frac{k_{c}}{2} \Gamma_{L}\left[\frac{k_{c}+2}{k_{c}} h\right],
$$

where $\Gamma_{L}[h]$ is related to the induced action eq. (3.21) through

$$
\Gamma[h]=\frac{c}{12} \Gamma_{L}[h] .
$$

The renormalized level $k_{c}$ is given by

$$
k_{c}=-\frac{1}{12}(25-c+\sqrt{(c-1)(c-25)})-2 .
$$




\section{Induced and effective $W_{3}$ gravity}

The action

$$
S[\phi]=\frac{1}{2 \pi} \int \partial \phi^{i} \bar{\partial} \phi^{i}, \quad i=1 \cdots n
$$

is invariant under

$$
\begin{aligned}
\delta \phi^{i} & =\epsilon \partial \phi^{i} \\
\delta \phi^{i} & =\lambda d^{i j k} \partial \phi^{j} \partial \phi^{k}
\end{aligned}
$$

provided

$$
\bar{\partial} \epsilon=\bar{\partial} \lambda=0 \text {. }
$$

Note that we introduced $d$ symbols $d^{i j k}$, which are totally symmetric. The commutator algebra of these transformations closes if

$$
d^{k(i j} d^{l) m k}=\delta^{(i j} \delta^{l) m}
$$

It is given by

$$
\begin{aligned}
& {\left[\delta\left(\epsilon_{1}\right), \delta\left(\epsilon_{2}\right)\right] \phi^{i}=\delta\left(\epsilon_{3}=\epsilon_{2} \partial \epsilon_{1}-\epsilon_{1} \partial \epsilon_{2}\right) \phi^{i}} \\
& {\left[\delta\left(\epsilon_{1}\right), \delta\left(\lambda_{2}\right)\right] \phi^{i}=\delta\left(\lambda_{3}=2 \lambda_{2} \partial \epsilon_{1}-\epsilon_{1} \partial \lambda_{2}\right) \phi^{i}} \\
& {\left[\delta\left(\lambda_{1}\right), \delta\left(\lambda_{2}\right)\right] \phi^{i}=\delta\left(\epsilon_{3}=-2\left(\lambda_{1} \partial \lambda_{2}-\lambda_{2} \partial \lambda_{1}\right) \partial \phi^{j} \partial \phi^{j}\right) \phi^{i}}
\end{aligned}
$$

The $\epsilon$ and $\lambda$ symmetries are the rigid $W_{3}$ symmetries of the scalar field theory. The currents associated with these symmetries are given by

$$
\begin{aligned}
T & =-\frac{1}{2} \partial \phi^{i} \partial \phi^{i} \\
W & =-\frac{i}{6} d^{i j k} \partial \phi^{i} \partial \phi^{j} \partial \phi^{k} .
\end{aligned}
$$

The classical current algebra (Poisson brackets, which means that only single contractions are made) is given by

$$
\begin{aligned}
T(x) T(y) & =2(x-y)^{-2} T(y)+(x-y)^{-1} \partial T(y)+\cdots \\
T(x) W(y) & =3(x-y)^{-2} W(y)+(x-y)^{-1} \partial W(y)+\cdots \\
W(x) W(y) & =(x-y)^{-2}(T T)(y)+\frac{1}{2}(x-y)^{-1} \partial(T T)(y)+\cdots
\end{aligned}
$$


The presence of composite operators in the current algebra is a direct reflection of the fact that the algebra of the transformations in eq. (4.5) has field dependent structure coefficients.

At the quantum level, the current algebra is more involved. It can be shown that the currents $T$ and $W$ in eq. (4.6) precisely generate the quantum $W_{3}$ algebra:

$$
\begin{aligned}
T(x) T(y)= & \frac{c}{2}(x-y)^{-4}+2(x-y)^{-2} T(y)+(x-y)^{-1} \partial T(y)+\cdots \\
T(x) W(y)= & 3(x-y)^{-2} W(y)+(x-y)^{-1} \partial W(y)+\cdots \\
W(x) W(y)= & \frac{c}{3}(x-y)^{-6}+2(x-y)^{-4} T(y)+(x-y)^{-3} \partial T(y) \\
& +(x-y)^{-2}\left[2 \beta \Lambda(y)+\frac{3}{10} \partial^{2} T(y)\right] \\
& +(x-y)^{-1}\left[\beta \partial \Lambda(y)+\frac{1}{15} \partial^{3} T(y)\right]+\cdots
\end{aligned}
$$

where

$$
\Lambda(x)=(T T)(x)-\frac{3}{10} \partial^{2} T(x)
$$

and

$$
\beta=\frac{16}{22+5 c},
$$

provided $n=2(c=2)$ and the $d$ symbols are traceless. If $n \neq 2$, the WIV OPE will contain an additional dimension 4 operator. Taking the OPE of this field with the $W$ current yields again new currents, etc. It is possible [34] to modify the currents $T$ and $W$ with terms containing higher derivatives of the $\phi^{i}$, so that the $W_{3}$ current algebra eq. (4.8) is satisfied with adjustable value of the central charge c.

In this paper, we will concentrate on pure $W_{3}$ matter systems, which by definition lead to currents $T(x)$ and $W(x)$ that satisfy the quantum $W_{3}$ algebra eq. (4.8) for some value of the central charge $c$. Further details of the microscopic structure of those matter systems will not be relevant for the coupling to $\mathrm{W}_{3}$ gravity. Though we will not make any restrictions on the value of the central charge, for concreteness one might wish to keep the $c=2$ example given above in mind. Note that in [10] several results were obtained for the scalar field model with an arbitrary number $n$ of scalar fields and no background charge couplings.

In the same spirit as the two preceding sections, we want to make the symmetries eq. (4.2) local. To do this we introduce the $W_{3}$ gauge fields $h_{--}$and $b_{-. .}$. For the 2-scalar field theory, where we define $B=b /(2 i)$, minimal coupling yields 
the action

$$
S=\frac{1}{2 \pi} \int d^{2} x\left(\partial \phi^{i} \partial \phi^{i}-h \partial \phi^{i} \partial \phi^{i}-\frac{2}{3} B \partial \phi^{i} \partial \phi^{i} \partial \phi^{k} d^{i j k}\right)
$$

which is gauge invariant if the gauge fields transform as

$$
\begin{aligned}
\delta h & =\bar{\partial} \epsilon+\epsilon \partial h-\partial \epsilon h+(\lambda \partial B-B \partial \lambda) \partial \phi^{i} \partial \phi^{i} \\
\delta B & =\bar{\partial} \lambda+2 \lambda \partial h-\partial \lambda h+\epsilon \partial B-2 \partial \epsilon B
\end{aligned}
$$

Let us now discuss the quantization of this theory. The induced action is defined as

$$
e^{-\Gamma_{\text {ind }}[h, b]}=\left\langle\exp -\frac{1}{\pi} \int d^{2} x[h(x) T(x)+b(x) W(x)]\right\rangle
$$

where $T$ and $W$ satisfy the OPE's in eq. (4.8). In the scalar field realization with $c=2$, where we have $b=2 i B$ and the currents are as in eq. (4.6), the induced action has a diagrammatic expansion given by

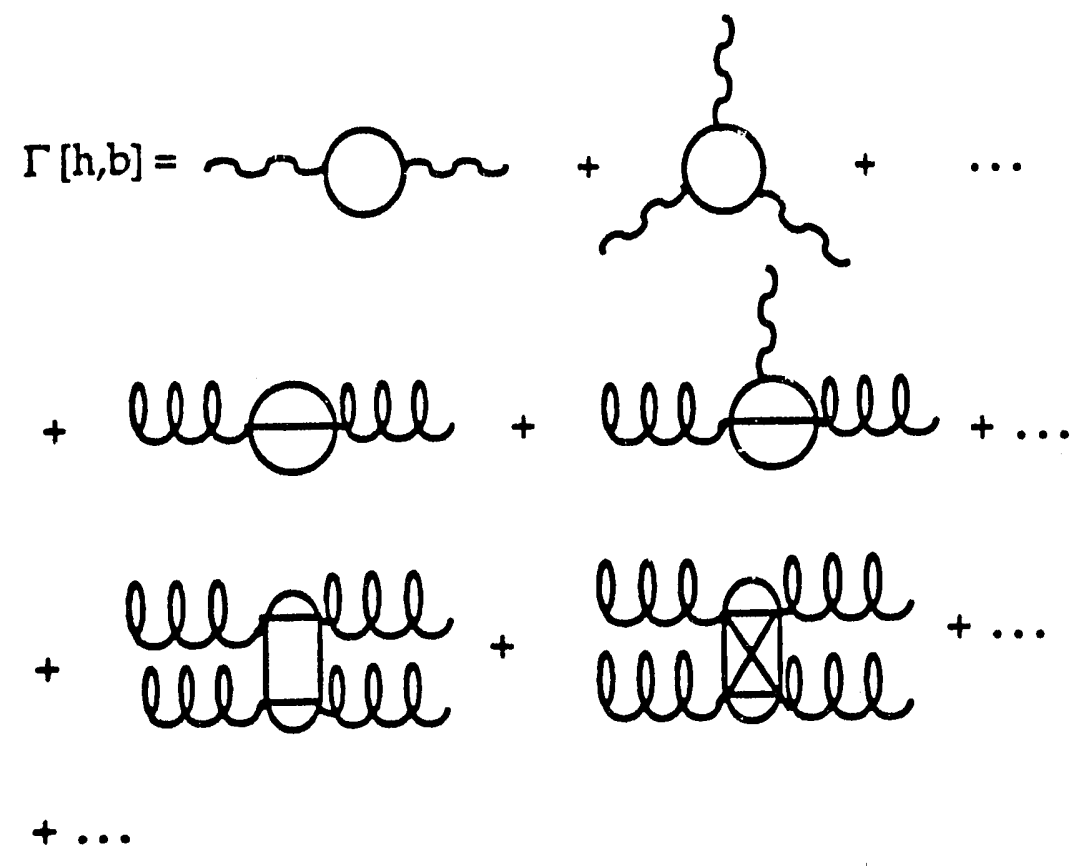


where the lines are

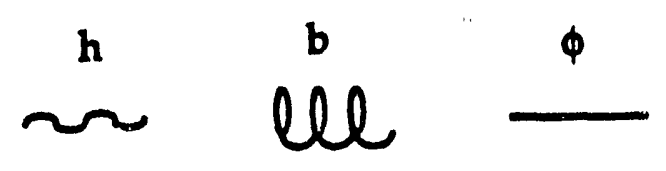

Note that diagrams with arbitrarily many loops appear. The $\epsilon$ anomaly arises in the same way as in the preceeding section: it comes from the 1-loop 2-point function. Indeed, under the variations

$$
\begin{aligned}
\delta h & =\bar{\partial} \epsilon+\epsilon \partial h-\partial \epsilon h \\
\delta b & =\epsilon \partial b-2 \partial \epsilon b,
\end{aligned}
$$

one has

$$
\delta_{\varepsilon} \Gamma_{\text {ind }}[h, b]=-\frac{c}{12 \pi} \int d^{2} x \in \partial^{3} h .
$$

The structure of the $\lambda$-anomalies turns out to be much more delicate. Requiring that all $\beta$-independent terms proportional to a single $u$ or $v$ in the $\lambda$ variation of $\Gamma[h, b]$ cancel, yields the first order $\lambda$ transformations

$$
\begin{aligned}
\delta h & =\frac{1}{30}\left(2 \lambda \partial^{3} b-3 \partial \lambda \partial^{2} b+3 \partial^{2} \lambda \partial b-2 \partial^{3} \lambda b\right) \\
\delta b & =\partial \lambda+2 \lambda \partial h-\partial \lambda h,
\end{aligned}
$$

and with these rules one has that

$$
\delta_{\lambda} \Gamma_{\text {ind }}[h, b]=-\frac{c}{360 \pi} \int d^{2} x \lambda \partial^{5} b-\frac{\beta}{\pi} \int d^{2} x \lambda(2 \partial b+b \partial) \Lambda_{\text {eff }} .
$$

Here

$$
\begin{aligned}
\Lambda_{\mathrm{eff}}(x)= & \left\langle\Lambda(x) \exp -\frac{1}{\pi} \int d^{2} y[h(y) T(y)+b(y) W(y)]\right\rangle / e^{-\Gamma[h, b]} \\
= & \frac{c^{2}}{144} u(x) u(x)-\frac{c \pi}{12} \lim _{y \rightarrow x}\left\{\frac{\delta u(x)}{\delta h(y)}-\frac{\partial_{x}{ }^{3}}{\partial_{x}} \delta(x-y)\right. \\
& \left.-2 \frac{\partial_{x}}{\partial_{x}} \delta(x-y) u(x)-\frac{1}{\partial_{x}} \delta(x-y) \partial u(x)\right\}-\frac{c}{40} \partial^{2} u(x),
\end{aligned}
$$


where

$$
u(x)=\frac{12 \pi}{c} \frac{\delta \Gamma_{\text {ind }}[h, b]}{\delta h(x)} .
$$

The first term in the r.h.s. of eq. (4.19) is precisely the anomaly one would expect from a spin-3 symmetry. However, there is a second term which is the result of the non-linearities in the $W_{3}$ algebra. As is clear from eq. (4.20), the regularization gives rise to subtle effects, which are subleading in $1 / c$.

In the limit $c \rightarrow \pm \infty$ one can neglect the regularization effects. Indeed one has then that $c^{-2} \Lambda_{\text {eff }}=\frac{1}{144} u^{2}$, and eq. (4.19) becomes $\delta \Gamma_{\text {ind }}[h, b]=-\frac{c}{360 \pi} \int d^{2} x \lambda \partial^{5} b-$ $\frac{c}{45 \pi} \int d^{2} x \lambda(2 \partial b+b \partial) u u$. In this limit we could reduce the $\lambda$ anomaly to the minimal one by adding an extra term to the $h$ transformation rule in eq. (4.18):

$$
\delta h=\frac{4}{15}(\lambda \partial b-b \partial \lambda) u .
$$

However, it turns out to be more natural to make a different choice for $\delta_{\text {extra }} h$ :

$$
\delta_{\text {extra }} h=\frac{8}{15}(\lambda \partial b-b \partial \lambda) u
$$

For this choice we have that $u$ and $v$ transform according to the operator product expansion in the limit $c \rightarrow \pm \infty$ (using that $\delta u(x) \sim \oint d y(\epsilon T+\lambda W)(y) u(x)$ and similar for $\delta v$ )

$$
\begin{aligned}
\delta u= & \partial^{3} \epsilon+\epsilon \partial u+2 \partial \epsilon u+\frac{1}{15} \lambda \partial v+\frac{1}{10} \partial \lambda v \\
\delta v= & \epsilon \partial v+3 \partial \epsilon v+\partial^{5} \lambda+\left(2 \lambda \partial^{3}+9 \partial \lambda \partial^{2}+15 \partial^{2} \lambda \partial+10 \partial^{3} \lambda\right) u \\
& +\delta(2 \partial \lambda+\lambda \partial) u u
\end{aligned}
$$

where

$$
v(x)=\frac{360 \pi}{c} \frac{\delta \Gamma_{\text {ind }}[h, b]}{\delta b(x)} .
$$

The algebra on the gauge fields and the effective currents is closed

$$
\begin{aligned}
{\left[\delta\left(\epsilon_{1}\right), \delta\left(\epsilon_{2}\right)\right]=\delta\left(\epsilon_{3}=\right.} & \left.\epsilon_{2} \partial \epsilon_{1}-\epsilon_{1} \partial \epsilon_{2}\right) \\
{\left[\delta\left(\epsilon_{1}\right), \delta\left(\lambda_{2}\right)\right]=\delta\left(\lambda_{3}=\right.} & \left.2 \lambda_{2} \partial \epsilon_{1}-\epsilon_{1} \partial \lambda_{2}\right) \\
{\left[\delta\left(\lambda_{1}\right), \delta\left(\lambda_{2}\right)\right]=\delta\left(\epsilon_{3}=\right.} & \frac{1}{30}\left(2 \partial^{3} \lambda_{1} \lambda_{2}-3 \partial^{2} \lambda_{1} \partial \lambda_{2}+3 \partial \lambda_{1} \partial^{2} \lambda_{2}-2 \lambda_{1} \partial^{3} \lambda_{2}\right) \\
& \left.+\frac{8}{15}\left(\lambda_{2} \partial \lambda_{1}-\lambda_{1} \partial \lambda_{2}\right) u\right)
\end{aligned}
$$


As we will see later on, it is precisely the choice eq. (4.23) for $\delta_{\text {extra }} h$ which will emerge from a constrained $S l(3, \mathbf{R})$ theory. With this choice, the $\lambda$ anomaly is not the minimal one:

$$
\delta_{\lambda} \Gamma_{\text {ind }}[h, b]=-\frac{c}{360 \pi} \int d^{2} x \lambda \partial^{5} b+\frac{c}{45 \pi} \int d^{2} x \lambda(2 \partial b+b \partial) u u .
$$

A useful check on this result is the analysis of the Wess-Zumino conditions for consistent anomalies, which are indeed satisfied (compare with our analysis in [10]).

Using the chain rule for $\delta \Gamma_{\text {ind }}$ and eqs. (4.16), (4.17), (4.18), (4.23) and (4.27) we find the final form of the Ward identities in the limit $c \rightarrow \pm \infty$ :

$$
\begin{aligned}
& \bar{\partial} u=D_{1} h+\left[\frac{1}{10} v \partial+\frac{1}{15}(\partial v)\right] b, \\
& \bar{\partial} v=[3 v \partial+(\partial v)] h+D_{2} b,
\end{aligned}
$$

where $D_{1}$ and $D_{3}$ are the 3 rd and 5th order Gelfand-Dickey operators given by

$$
\begin{aligned}
& D_{1}=\partial^{3}+2 u \partial+u^{\prime}, \\
& D_{2}=\partial^{5}+10 u \partial^{3}+15 u^{\prime} \partial^{2}+9 u^{\prime \prime} \partial+2 u^{\prime \prime \prime}+16 u^{2} \partial+16 u u^{\prime},
\end{aligned}
$$

and the primes denote $\partial$.

This is the situation for $c \rightarrow \pm \infty$. Solving eqs. (4.28) for $\Gamma[h, b]$ yields the induced action in this limit, which we will denote by $\Gamma_{\infty}[h, b]$. Later in this section we will explicitely compute this action, using a constrained $S l(3, \mathbf{R})$ WZIV model. Before coming to this, let us first comment on the finite $c$ corrections to the Ward identities. The induced action of $W_{3}$ gravity can be expanded in even powers of the field $b$. The full result for the $b$-independent terns in $\Gamma_{\text {ind }}[h, b]$ (1-loop diagrams) is given by

$$
\Gamma_{\text {ind }}^{[0]}[h, b]=\frac{c}{24 \pi} \int d^{2} x \partial^{2} h \frac{1}{\partial} \frac{1}{1-h \frac{\partial}{\partial}} \partial h .
$$

The exact result for the terms quadratic in $b$ (2-loop diagrams) reads

$$
\Gamma_{\text {ind }}^{[2]}[h, b]=\frac{c}{720 \pi} \int \partial Y \bar{\nabla} Y,
$$

where $\bar{\nabla}=\bar{\partial}-h \partial$ and the scalar field $Y$ is given by

$$
Y=\frac{1}{\bar{\nabla}}\left[\partial+\left(\frac{\partial}{\partial} \frac{1}{1-h \frac{\partial}{\partial}} \partial h\right)\right]\left[\partial b+2 b\left(\frac{\partial}{\partial} \frac{1}{1-h \frac{\partial}{\partial}} \partial h\right)\right] .
$$


The leading ' 3 -loop diagrams' (i.e., the terms with four $b$ fields and no external $h$ fields) is given by

$$
\Gamma_{\text {ind }}^{[1]}[h=0, b]=\frac{c}{60 \cdot 6 !} \frac{1}{\pi}[I]+\frac{2 \beta c}{5 \cdot 7 !} \frac{1}{\pi}[I I],
$$

where $[I]$ and $[I I]$ are given by

$$
\begin{aligned}
& {[I]=\int d^{2} x\left(2 b \frac{\partial^{3}}{\partial} b-3 \partial b \frac{\partial^{2}}{\partial} b+3 \partial^{2} b \frac{\partial}{\partial} b-2 \partial^{3} b \frac{1}{\partial} b\right) \frac{1}{\partial}\left(2 b \frac{\partial^{6}}{\partial} b+3 \partial b \frac{\partial^{5}}{\partial} b\right),} \\
& {[I I]=\int d^{2} x\left(b \frac{\partial}{\partial} b-\partial b \frac{1}{\partial} b\right) \frac{1}{\partial} Q_{b b}^{8},}
\end{aligned}
$$

where

$$
Q_{b b}^{8}=b \frac{\partial^{8}}{\partial} b+6 \partial b \frac{\partial^{\prime \prime}}{\partial{ }^{\prime}} b+14 \partial^{2} b \frac{\partial^{6}}{\partial} b+14 \partial^{3} b \frac{\partial^{5}}{\partial} b .
$$

Since the quantity $\beta c$ is of order 1 for: large $c$, one sees here explicitely the onset of a $1 / c$ expansion for the induced action as in eq. (1.2). As such, the Ward identities will be modified for finite $c$. Indeed, from eq. (4.33) we see that structure $[I]$ gives the three-loop contribution to eq. (4.28) while structure $[I I]$ is subleading in the $1 / c$ expansions and modifies the large $c$ Ward identities for the induced action:

$$
\begin{aligned}
\bar{\partial} u= & D_{1} h+\frac{1}{30}[3 v \partial \hat{a}+2(\partial v)] b, \\
\bar{\partial} v= & {[3 v \partial+(\partial v)] h+D_{2} b } \\
& +\frac{4 \beta}{35}\left(2 \partial b \frac{1}{\partial} Q_{b b}^{8}+b \frac{\partial}{\partial} Q_{b b}^{8}\right)+\mathcal{O}\left(b^{3} h^{\geq 1}, b^{5}, \ldots\right) .
\end{aligned}
$$

We will now explicitely compute $\Gamma_{\text {ind }}[h, b]$ in the limit $c \rightarrow \pm \infty$, denoted by $\Gamma_{\infty}[h, b]$. We follow essentially the same method as in the previous section, but this time we start from an $S l(3, R)$ model. Our analysis closely follows that in [14]; note that some partial results were also obtained in $[35,36,37]$.

We choose the following basis for $\operatorname{Sl}(3, \mathrm{R})$ :

$$
\begin{aligned}
& T_{1} \equiv e_{11}-e_{22}, \quad T_{2} \equiv e_{22}-e_{33} \\
& T_{+1} \equiv e_{12}, \quad T_{+2} \equiv e_{23}, \quad T_{+3} \equiv e_{13} \\
& T_{-1} \equiv e_{21}, \quad T_{-2} \equiv e_{32}, \quad T_{-3} \equiv e_{31},
\end{aligned}
$$

where $e_{i j}$ are $3 \times 3$ matrices. $\left(e_{i j}\right)_{k l}=\delta_{i k} \delta_{j l}$. We impose the following constraints

$$
u \equiv\left(\begin{array}{ccc}
0 & u^{+1} & u^{+3} \\
1 & 0 & 0 \\
0 & 1 & 0
\end{array}\right) .
$$


Six of the eight components of the Ward identities $\bar{\partial} u-[A, u]=\partial A$ can be used to express the fields $A^{1}, A^{2}, A^{ \pm 2}, A^{+1}$ and $A^{+3}$ in terms of $A^{-1}, A^{-3}$ and their conjugates $u^{+1}, u^{+3}$

$$
\begin{aligned}
A^{-2} & =-\partial A^{-3}+A^{-1} \\
A^{1} & =-\frac{1}{3}\left(\partial^{2} A^{-3}-3 \partial A^{-1}-u^{+1} A^{-3}\right) \\
A^{2} & =-\frac{2}{3}\left(\partial^{2} A^{-3}-\frac{3}{2} \partial A^{-1}-u^{+1} A^{-3}\right) \\
A^{+1} & =\frac{1}{3}\left(\partial^{3} A^{-3}-3 \partial^{2} A^{-1}-\partial\left(u^{+1} A^{-3}\right)\right)+u^{+1} A^{-1}+u^{+3} A^{-3} \\
A^{+2} & =\frac{2}{3}\left(\partial^{3} A^{-3}-\frac{3}{2} \partial^{2} A^{-1}-\partial\left(u^{+1} A^{-3}\right)\right)+u^{+3} A^{-3} \\
A^{+3} & =\frac{2}{3}\left(\partial^{4} A^{-3}-\frac{3}{2} \partial^{3} A^{-1}-\partial^{2}\left(u^{+1} A^{-3}\right)\right)+\partial\left(u^{+3} A^{-3}\right)+u^{+3} A^{-1} .
\end{aligned}
$$

The remaining two identities are non-trivial and read

$$
\begin{aligned}
-2 \partial^{3} \tilde{A}^{-1}= & \left(\bar{\partial}-2 \partial \tilde{A}^{-1}-\tilde{A}^{-1} \partial\right) u^{+1}-\left(2 . A^{-3} \partial+3 \partial A^{-3}\right) \tilde{u}^{+3} \\
\frac{1}{6} \partial^{5} A^{-3}= & \frac{1}{12}\left(2 A^{-3} \partial^{3}+9 \partial A^{-3} \partial^{2}+15 \partial^{2} A^{-3} \partial+10 \partial^{3} A^{-3}\right) u^{+1} \\
& -\frac{1}{3} \partial\left(u^{+1} u^{+1}\right) A^{-3}-\frac{2}{3} u^{+1} u^{+1} \partial A^{-3} \\
& +\left(\bar{\partial}-\tilde{A}^{-1} \partial-3 \partial \tilde{A}^{-1}\right) \tilde{u}^{+3}
\end{aligned}
$$

where

$$
\tilde{A}^{-1}=A^{-1}-\frac{1}{2} \partial A^{-3}, \quad \tilde{u}^{+3}=u^{+3}-\frac{1}{2} \partial u^{+1} .
$$

Comparing eq. (4.40) with the $W_{3}$ Ward identities for $c \rightarrow \pm \infty$ in eq. (4.28), one finds that they are identical if one identifies

$$
\begin{aligned}
u=-\frac{1}{2} u^{+1}, & v & =-15 \gamma \tilde{u}^{+3} \\
h=\tilde{A}^{-1}, & b & =\gamma^{-1} A^{-3}
\end{aligned}
$$

and puts $\gamma^{2}=-2 / 5$

The action $\Gamma_{\infty}[h, b]$ for induced $W_{3}$ gravity can now be obtained from the $S l(3, \mathbf{R})$ action in exactly the same way as we obtained the action $\Gamma[h]$ for induced 
pure gravity from $S l(2, \mathbf{R})$. We find

$$
\Gamma_{\infty}[h, b]=-\Gamma_{W Z W}\left[A^{-1}, A^{-3}\right]+\frac{k}{\pi} \int d^{2} x\left(u^{+1} A^{-1}+2 u^{+3} A^{-3}\right)
$$

where we should put

$$
k=\frac{c}{24} .
$$

The latter identification agrees with the leading term of a $W_{3} \mathrm{KPZ}$ formula [32, 9]. We see that the induced action for $W_{3}$ gravity takes the form of the Legendre tiansform of a constrained WZW model.

In order to obtain an explicit form for the action we introduce a Gaussian parametrization for $S l(3, \mathbf{R})$ :

$$
g=\left(\begin{array}{ccc}
1 & \phi_{1} & \phi_{3} \\
0 & 1 & \phi_{2} \\
0 & 0 & 1
\end{array}\right)\left(\begin{array}{ccc}
\lambda_{1}^{-1} & 0 & 0 \\
0 & \lambda_{2}^{-1} & 0 \\
0 & 0 & \lambda_{1} \lambda_{2}
\end{array}\right)\left(\begin{array}{ccc}
1 & 0 & 0 \\
f_{2} & 1 & 0 \\
f_{3} & f_{1} & 1
\end{array}\right)
$$

From $u=\partial g g^{-1}$, we can solve the constraints eq. (4.38),

$$
\begin{aligned}
f_{2} & =\frac{\partial f_{3}}{\partial f_{1}} \\
\phi_{1} & =-\frac{1}{3}\left(\partial f_{1}\right)^{-1} \partial^{2} f_{1}-\frac{2}{3}\left(\partial\left(\frac{\partial f_{3}}{\partial f_{1}}\right)\right)^{-1} \partial^{2}\left(\frac{\partial f_{3}}{\partial f_{1}}\right) \\
\phi_{2} & =-\frac{1}{3} \frac{\partial^{3} f_{3} \partial f_{1}-\partial f_{3} \partial^{3} f_{1}}{\partial^{2} f_{3} \partial f_{1}-\partial f_{3} \partial^{2} f_{1}} \\
\phi_{3} & =\partial \phi_{2}+\phi_{2}{ }^{2} \\
\lambda_{1}{ }^{3} & =\left(\partial\left(\frac{\partial f_{3}}{\partial f_{1}}\right)\right)^{-2}\left(\partial f_{1}\right)^{-1} \\
\lambda_{2}{ }^{3} & =\partial\left(\frac{\partial f_{3}}{\partial f_{1}}\right)\left(\partial f_{1}\right)^{-1}
\end{aligned}
$$

From $A=\bar{\partial} g g^{-1}$ and eq. (4.42) we obtain

$$
\begin{aligned}
h & =\frac{\bar{\partial}\left(\frac{\partial f_{3}}{\partial f_{1}}\right)}{\partial\left(\frac{\partial f_{3}}{\partial f_{1}}\right)}-\frac{\gamma}{3} \frac{\partial^{3} f_{3} \partial f_{1}-\partial f_{3} \partial^{3} f_{1}}{\partial^{2} f_{3} \partial f_{1}-\partial f_{3} \partial^{2} f_{1}} b-\frac{\gamma}{2} \partial b \\
b & =\gamma^{-1} \frac{\left(\bar{\partial} f_{3} \partial f_{1}-\bar{\partial} f_{1} \partial f_{3}\right)}{\left(\partial^{2} f_{3} \partial f_{1}-\partial^{2} f_{1} \partial f_{3}\right)} .
\end{aligned}
$$


The effective currents $u$ and $v$ are

$$
\begin{aligned}
& u=-\frac{1}{2}\left[\partial\left(2 \frac{\partial \lambda_{1}}{\lambda_{1}}+\frac{\partial \lambda_{2}}{\lambda_{2}}\right)+\left(\frac{\partial \lambda_{1}}{\lambda_{1}}\right)^{2}+\left(\frac{\partial \lambda_{2}}{\lambda_{2}}\right)^{2}+\left(\frac{\partial \lambda_{1}}{\lambda_{1}}\right)\left(\frac{\partial \lambda_{2}}{\lambda_{2}}\right)\right] \\
& v=-15 \gamma\left[\frac{1}{2} \partial^{2}\left(\frac{\partial \lambda_{2}}{\lambda_{2}}\right)+\left(\frac{3}{2} \frac{\partial \lambda_{1}}{\lambda_{1}}+\frac{\partial \lambda_{2}}{\lambda_{2}}\right) \partial\left(\frac{\partial \lambda_{2}}{\lambda_{2}}\right)\right. \\
&\left.+\frac{1}{2}\left(\frac{\partial \lambda_{2}}{\lambda_{2}}\right) \partial\left(\frac{\partial \lambda_{1}}{\lambda_{1}}\right)+\left(\frac{\partial \lambda_{1}}{\lambda_{1}}+\frac{\partial \lambda_{2}}{\lambda_{2}}\right)\left(\frac{\partial \lambda_{1}}{\lambda_{1}}\right)\left(\frac{\partial \lambda_{2}}{\lambda_{2}}\right)\right] .
\end{aligned}
$$

A different parametrization, which stays closer to the Polyakov parametrization, is given by

$$
\begin{aligned}
& f_{1}=f \\
& f_{3}=\frac{1}{2} f^{2}+g .
\end{aligned}
$$

In linearized form, this parametrization was already found in [10]. In these variables one has that

$$
\begin{aligned}
h= & \left(1+\partial^{2} g\right)^{-1}\left(\frac{\bar{\partial} f}{\partial f}+\frac{1}{\partial f} \bar{\partial} \phi g\right) \\
& -\frac{\gamma}{3} \partial\left(\ln \left[(\partial f)^{3}\left(1+\partial^{2} g\right)\right]\right) b-\frac{\gamma}{2} \partial b \\
b= & \frac{1}{\gamma} \frac{\left(\bar{\partial}-\frac{\partial f}{\partial f} \partial\right) g}{(\partial f)^{2}\left(1+\partial^{2} g\right)}
\end{aligned}
$$

where

$$
\not \partial=(\partial f)^{-1} \partial
$$

Pure gravity now simply corresponds to putting $g=0$.

We can now substitute the Gaussian decomposition into the action in (4.43). There is now no cancellation between the binetic term of the WZW model and the $u A$ correction term and one finds the following surprisingly simple expression for the induced action

$$
\begin{aligned}
& \Gamma_{\infty}\left[h\left(f_{1}, f_{3}\right), b\left(f_{1}, f_{3}\right)\right]= \\
& \begin{aligned}
-\frac{c}{48 \pi} \int d^{2} x & \left\{\frac{\partial\left(\frac{\partial f_{3}}{\partial f_{1}}\right)}{\partial\left(\frac{\partial f_{3}}{\partial f_{1}}\right)} \partial\left(\frac{\partial \lambda_{1}}{\lambda_{1}}\right)+\frac{\partial f_{1}}{\partial f_{1}} \partial\left(\frac{\partial \lambda_{1}}{\lambda_{1}}+\frac{\partial \lambda_{2}}{\lambda_{2}}\right)\right. \\
& +\frac{\partial f_{3} \partial f_{1}-\bar{\partial} f_{1} \partial f_{3}}{\partial^{2} f_{3} \partial f_{1}-\partial^{2} f_{1} \partial f_{3}}\left[\frac{\partial \lambda_{2}}{\lambda_{2}} \partial\left(\frac{\partial \lambda_{1}}{\lambda_{1}}\right)-\frac{\partial \lambda_{1}}{\lambda_{1}} \partial\left(\frac{\partial \lambda_{2}}{\lambda_{2}}\right)\right.
\end{aligned}
\end{aligned}
$$




$$
\left.\left.-\left(\frac{\partial \lambda_{1}}{\lambda_{1}}\right)\left(\frac{\partial \lambda_{2}}{\lambda_{2}}\right)\left(\frac{\partial \lambda_{1}}{\lambda_{1}}+\frac{\partial \lambda_{2}}{\lambda_{2}}\right)\right]\right\} .
$$

By using the expressions in eq. (4.46), this can be further reduced to an expression in terms of $f_{1}$ and $f_{3}$ only. An expression of $\Gamma$ in terms of $h$ and $b$ seems hard to obtain, as it is not clear to us how the relations (4.47) or (4.50) can be inverted explicitly.

From $\delta u=\partial \eta+[\eta, u]$, we can express $\eta^{1}, \eta^{2}, \eta^{ \pm 2}, \eta^{+1}$ and $\eta^{+3}$ in terms of $\eta^{-1}, \eta^{-3}, u^{+1}$ and $u^{+3}$. The result can, of course, immediately be read off from the fact that $\bar{\partial} u-[A, u]=\partial A$ and $\delta u-[\eta, u]=\partial \eta$ have a similar structure. For the transformation rules of the effective currents $u$ and $v$ we find

$$
\begin{aligned}
\delta u= & \partial^{3} \epsilon+\epsilon \partial u+2 \partial \epsilon u+\frac{1}{15} \lambda \partial v+\frac{1}{10} \partial \lambda v, \\
\delta v= & \epsilon \partial v+3 \partial \epsilon v+\partial^{5} \lambda+\left(2 \lambda \partial^{3}+9 \partial \lambda \partial^{2}+15 \partial^{2} \lambda \partial+10 \partial^{3} \lambda\right) u \\
& +8(2 \partial \lambda+\lambda \partial) u u,
\end{aligned}
$$

where

$$
\begin{aligned}
\epsilon & =\eta^{-1}-\frac{1}{2} \partial \eta^{-3} \\
\lambda & =\gamma^{-1} \eta^{-3}
\end{aligned}
$$

which agrees with eqs. (1.24). Combining this with $\delta A=\bar{\partial} \eta+[\eta, A]$ and eqs. (4.42) we obtain

$$
\begin{aligned}
\delta h= & \bar{\partial} \epsilon+\epsilon \partial h-\partial \epsilon h+\frac{1}{30}\left(2 \lambda \partial^{3}-3 \partial \lambda \partial^{2}+3 \partial^{2} \lambda \partial-2 \partial^{3} \lambda\right) b \\
& +\frac{8}{15}(\lambda \partial b-b \partial \lambda) u \\
\delta b= & \epsilon \partial b-2 \partial \epsilon b+\bar{\partial} \lambda+2 \lambda \partial h-\partial \lambda h
\end{aligned}
$$

in agreement with (4.18) and (4.23). Combining the solution of the constraints with the fact that $\left(\delta g g^{-1}\right)=\eta$ yields

$$
\begin{aligned}
& \delta f_{1}=\epsilon \partial f_{1}+\gamma \lambda \partial^{2} f_{1}-\frac{\gamma}{2} \partial \lambda \partial f_{1}-\frac{2 \gamma}{3} \lambda \partial f_{1} \partial \ln \left[\left(\partial^{2} f_{3} \partial f_{1}-\partial^{2} f_{1} \partial f_{3}\right)\right] \\
& \delta f_{3}=\epsilon \partial f_{3}+\gamma \lambda \partial^{2} f_{3}-\frac{\gamma}{2} \partial \lambda \partial f_{3}-\frac{2 \gamma}{3} \lambda \partial f_{3} \partial \ln \left[\left(\partial^{2} f_{3} \partial f_{1}-\partial^{2} f_{1} \partial f_{3}\right)\right] .(4
\end{aligned}
$$

After the change of variables eq. (4.49), we find

$$
\delta f=c \partial f-\gamma \lambda \partial^{2} f-\frac{1}{2} \gamma \partial \lambda \partial f-\frac{2}{3} \gamma \lambda \frac{\partial^{3} g}{1+\partial^{2} g}
$$




$$
\begin{aligned}
\delta g= & \epsilon \partial g+\gamma \lambda(\partial f)^{2}+\gamma \lambda \partial^{2} g-\frac{1}{2} \gamma \partial \lambda \partial g \\
& -\frac{2}{3} \gamma \lambda \partial g\left(3 \frac{\partial^{2} f}{\partial f}+\frac{\partial \partial^{2} g}{1+{\partial^{2} g}^{2}}\right) .
\end{aligned}
$$

We will now study the quantization of the $W_{3}$ gauge fields which leads to the effective action. At first sight, the presence of subleading terms in the induced action seems to cause problems. However, we found evidence, which we will show in a moment, that the subleading terms in the induced action donot appear in the effective action, since they are cancelled by further terms that arise from quantum loops of the gauge fields $h$ and $b$. We will argue that the effective 1PI action will be equal, up to multiplicative renormalizations, to the leading term in the $1 / c$ expansion of the induced action.

Before we proceed, we first introduce a ( $c$-independent) reference functional $\Gamma_{L}[h, b]$, which is such that the leading terms induced action can be written as

$$
\Gamma_{\text {ind }}[h, b]=\frac{c}{12} \Gamma_{L}[h, b]+\cdots,
$$

where the dots indicate terms of order $1,1 / c$, etc. The first few terms in $\Gamma_{L}[h, b]$ are

$$
\Gamma_{L}[h, b]=\frac{1}{2 \pi} \int d^{2} x h \frac{\partial^{3}}{\bar{\partial}} h+\frac{1}{60 \pi} \int d^{2} x b \frac{\partial^{5}}{\bar{\partial}} b+\cdots .
$$

We have that $\Gamma_{L}[h, b]$ satisfies the Ward identities eq. (4.28) with

$$
u=\pi \frac{\delta}{\delta h} \Gamma_{L}, \quad v=30 \pi \frac{\delta}{\delta b} \Gamma_{L} .
$$

A related functional $W_{L}[u, v]$ is obtained from $\Gamma_{L}[h, b]$ by a Legendre transformation

$$
W_{L}[u, v]=\Gamma_{L}[h(u, v), b(u, v)]-\frac{1}{\pi} \int d^{2} x\left(h u+\frac{1}{30} b v\right),
$$

where $h(u, v)$ and $b(u, v)$, which we denote by $h_{L}(u, v)$ and $b_{L}(u, v)$ for later reference, are determined through the relations in (4.60) and we have that

$$
h_{L}(u, v)=-\pi \frac{\delta}{\delta u} W_{L}, \quad b_{L}(u, v)=-30 \pi \frac{\delta}{\delta v} W_{L} .
$$

The induced action can now be written in terms of these reference functionals

$$
\Gamma_{\text {ind }}[h, b]=\frac{c}{12} \Gamma_{L}[h, b]
$$




$$
\begin{aligned}
& +\frac{32}{25 \cdot 7 !} \frac{1}{\pi}[I I]+\mathcal{O}\left(b^{4} h^{\geq 1}, b^{6}, \ldots\right) \\
& +\mathcal{O}\left(\frac{1}{c}\right),
\end{aligned}
$$

where the consecutive lines are the terms of order $c, 1$ and $\mathcal{O}(1 / c)$, respectively.

The generating functional $W[t, w]$ of connected Green's functions is defined by

$$
e^{-W[t, w]}=\int[d h][d b] e^{-\Gamma_{\text {ind }}[h, b]+\frac{1}{\pi} \int d^{2} x(h t+b w)} .
$$

The Legendre transform of $W[t, w]$ yields the 1PI or effective action. In tirms of diagrams, the complete perturbative evaluation of $W[t, w]$ involves two independent loop expansions, one with matter loops due to the path integral over $\phi$ and the second with gauge field loops due to the integral over $h$ and $b$. The net result can be analyzed as follows in terms of a $1 / c$ expansion for large $c$ (which is the weak coupling regime).

In the same spirit as in section 2, we approximate the path integral (4.64) by the saddle-point contribution. This leads to the leading term in $W[t, w]$, which is simply the Legendre transform of the induced action. This tree result should then be corrected by further terms coming from diagrams with $h$ and $b$ loops. We now observe that the kinetic terms for $h$ and $b$ in the induced action are proportional to $c$, such that $1 / c$ plays the role of Planck's constant in the path integral (4.64), while the interaction terms in the induced action are of order $c$ or subleading by extra powers of $1 / c$ (see (4.30), (4.31), (4.33) and (4.63)). From this it follows that the the loop-corrections to the saddle-point result are suppressed by a strictly positive power of $1 / c$ as compared to the leading terms in the saddle-point result.

The $1 / c$ expansion of the induced action $\Gamma_{\text {ind }}[h, b]$ in (4.63) leads to a $1 / c$ expansion of the saddle-point contribution to $W[t, w]$ when the latter is viewed as a function of $t / c$ and $w / c$, i.e.,

$$
\begin{aligned}
W[t, w]= & -\frac{6 c}{\pi} \int d^{2} x\left(\frac{t}{c}\right) \frac{\bar{\partial}}{\partial^{3}}\left(\frac{t}{c}\right)-\frac{180 c}{\pi} \int d^{2} x\left(\frac{w}{c}\right) \frac{\bar{\partial}}{\partial^{5}}\left(\frac{w}{c}\right)+\ldots \\
& +\frac{32}{25 \cdot \bar{\tau} !} \frac{1}{\pi}[I I]\left[b \rightarrow \frac{\partial}{\partial^{5}}\left(\frac{360}{c} w\right)\right]+\ldots
\end{aligned}
$$

Through the orders $c$ and $c^{0}$, this saddle-point contrilution can be written as

$$
\Gamma_{\text {ind }}[h, b]-\frac{1}{\pi} \int d^{2} x(h t+b w)
$$


with $h(t, w)=h_{L}\left(\frac{12}{c} t, \frac{360}{c} w\right)$ and $b(t, w)=b_{L}\left(\frac{12}{c} t, \frac{360}{c} w\right)$, see (4.62). Using the relation (4.61), one finds that the leading term $\frac{c}{12} \Gamma_{L}[h, b]$ in $\Gamma_{\text {ind }}[h, b]$ leads to the term $\frac{c}{12} W_{L}\left[\frac{12}{c} t, \frac{360}{c} w\right]$ in $W[t, w]$.

We shall now concentrate on the leading $c^{0}$ terms in the full expression for $W[t, w]$. These come from two sources: (i) the explicit terms in the second line of the induced action (4.63), evaluated at the saddle point, and (ii) the 1-loop corrections in the second path-integral (4.64). The latter can be computed by standard determinant techniques, as was demonstrated by Polyakov (unpublished, see [31]) for the case of pure gravity. The relevant determinant is the determinant of the matrix of second derivatives of the induced action $\Gamma_{\text {ind }}[h, b]$ with respect to $h$ and $b$, evaluated at the saddle-point. In here we may again replace $h$ and $b$ by their leading parts $h_{L}\left(\frac{12}{c} t, \frac{360}{c} w\right)$ and $b_{L}\left(\frac{12}{c} t, \frac{360}{c} w\right)$ (we will assume that this has been done in what follows). One finds the following contribution to $W[t, w]$

$$
W[t, w ; 1-\text { loop }]=\frac{1}{2} \ln \operatorname{det}\left(\begin{array}{cc}
\frac{\delta u}{\delta h} & \frac{\delta v}{\delta h} \\
\frac{\delta u}{\delta b} & \frac{\delta v}{\delta b}
\end{array}\right) .
$$

Since we are only interested in the terms independent of $c$, we can in this 1 loop calculation replace the full induced action by the leading term $\frac{c}{12} \Gamma_{L}[h, b]$. This means that we can use the Ward identities (4.28) to calculate the functional derivatives of $u$ and $v$ w.r.t. $h$ and $b$. It turas out that the determinant factorizes and one obtains

$$
\begin{aligned}
& W[t, w ; 1-\text { loop }]= \\
& -\frac{1}{2} \ln \operatorname{det}\left(\begin{array}{cc}
\bar{\nabla}_{2} & -\frac{1}{10}(\partial b)-\frac{1}{15} b \partial \\
L & \bar{\nabla}_{3}
\end{array}\right)+\frac{1}{2} \ln \operatorname{det}\left(\begin{array}{cc}
D_{1} & \frac{1}{10} v \partial+\frac{1}{15}(\partial v) \\
3 v \partial+(\partial v) & D_{2}
\end{array}\right),
\end{aligned}
$$

where $\bar{\nabla}_{j}=\bar{\partial}-h \partial-j(\partial h)$ and the operator $L$ is given by

$$
L=-\left(10 \partial^{3} b+15 \partial^{2} b \partial+9 \partial b \partial^{2}+2 b \partial^{3}+32 u \partial b+16 b \partial u+16 b u \partial\right) .
$$

Our task is now to evaluate these two fundamental determinants. The basic covariant differential operators $\bar{\nabla}_{j}$ and $D_{j}$, which can be defined for all integers $j$, can be expressed in terms of the reference functionals $\Gamma_{L}^{\text {grav }}[h]$ and $W_{L}^{\text {grav }}[u]$ defined by the relations

$$
\begin{gathered}
u=\pi \frac{\delta}{\delta h} \Gamma_{L}^{\mathrm{grav}}, \quad h=-\pi \frac{\delta}{\delta u} W_{L}^{\mathrm{grav}}, \\
\bar{\partial} u=D_{1} h,
\end{gathered}
$$


according to

$$
\begin{aligned}
& \ln \operatorname{det} \nabla_{j}=\frac{\left(6 j^{2}-6 j+1\right)}{6} \Gamma_{L}^{\mathrm{grav}}[h], \\
& \ln \operatorname{det} D_{j}=-\frac{j(2 j+1)(2 j+2)}{6} W_{L}^{\text {grav }}[u] .
\end{aligned}
$$

One would therefore expect that, at least to leading order, similar formulas can be established for our determinants of $2 \times 2$ operators, this time in terms of the $W_{3}$ reference functionals $\Gamma_{L}[h, b]$ and $W_{L}[u, v]$. In order to actually compute the determinants, we shall use a representation in terms of auxiliary b-c systems and make use, once more, of current algebra.

If we define two b-c systems by the following actions,

$$
\begin{aligned}
S\left[b_{1,2}, c_{1,2} ; h, b\right] & =\frac{1}{\pi} \int\left(\begin{array}{ll}
b_{1} & b_{2}
\end{array}\right)\left(\begin{array}{cc}
\bar{\nabla}_{2} & -\frac{1}{10}(\partial b)-\frac{1}{13} b \partial \\
L & \bar{\nabla}_{3}
\end{array}\right)\left(\begin{array}{l}
c_{1} \\
c_{2}
\end{array}\right), \\
S\left[B_{1,2}, C_{1,2} ; u, v\right] & =\frac{1}{\pi} \int\left(\begin{array}{ll}
B_{1} & B_{2}
\end{array}\right)\left(\begin{array}{cc}
D_{1} & \frac{1}{10} v \partial+\frac{1}{15}(\partial v) \\
3 v \partial+(\partial v) & D_{2}
\end{array}\right)\left(\begin{array}{l}
C_{1} \\
C_{2}
\end{array}\right),
\end{aligned}
$$

then the determinants in (4.68) are just the partition functions of these auxiliary field theories. Using operator techniques, one can explicitely evaluate these partition functions [15]. The results read

$$
\begin{aligned}
& \ln \operatorname{det}\left(\begin{array}{cc}
\bar{\nabla}_{2} & -\frac{1}{10}(\partial b)-\frac{1}{15} b \partial \\
L & \bar{\nabla}_{3}
\end{array}\right)= \\
& \frac{50}{6} \Gamma_{L}[h, b]+\frac{272}{5 \pi c} \int w(h, b) b+\frac{64}{25 \cdot 7 !} \frac{1}{\pi}[I I]+\ldots \\
& \ln \operatorname{det}\left(\begin{array}{cc}
D_{1} & \frac{1}{10} v \partial+\frac{1}{15}(\partial v) \\
3 v \partial+(\partial v) & D_{2}
\end{array}\right)=-12 W_{L}[u, v]+\ldots
\end{aligned}
$$

These expressions are exact through the orders $h^{n}, b^{2}, b^{2} h$ and $b^{4}$.

We are now ready to combine the above results into an expression for the effective action, which is exact through the orders $h^{n}, b^{2}, b^{2} h$ and $b^{4}$ in the leading $1 / c$ correction to the saddle-point result. To our great satisfaction, we find that the explicit non-local structure $[I I]$ precisely cancels between the induced action and the determinant corrections. The remaining terms are

$$
W[t, w]=\frac{c}{12} W_{L}\left[\frac{12}{c} t, \frac{360}{c} w\right]-6 W_{L}\left[\frac{12}{c} t, \frac{360}{c} w\right]-\frac{50}{12} \Gamma_{L}[h, b]-\frac{1}{\pi c} \frac{272}{10} \int w b+\ldots
$$


Once more using the saddle-point equations, we can rewrite this as

$$
W[t, w]=\frac{c}{12}\left(1-\frac{122}{c}+\ldots\right) W_{L}\left[\frac{12}{c}\left(1+\frac{50}{c}+\ldots\right) t, \frac{360}{c}\left(1+\frac{386}{5 c}+\ldots\right) w\right] .
$$

We thus find that the computed result for $W[t, w]$ can be summarized by the simple formula (4.76). We now propose that the exact, all-order result for the functional $W[t, w]$ can be gotten by simply completing the $1 / c$ expansions indicated by the dots in (4.76). This leads to the formula

$$
W[t, w]=2 k_{c} W_{L}\left[Z^{(t)} t, Z^{(w)} w\right]
$$

where $k_{c}, Z^{(t)}$ and $Z^{(w)}$ are functions of $c$ that allow the $1 / c$ expansions

$$
\begin{aligned}
k_{c} & =\frac{c}{24}\left(1-\frac{122}{c}+\ldots\right) \\
Z^{(t)} & =\frac{12}{c}\left(1+\frac{50}{c}+\ldots\right) \\
Z^{(w)} & =\frac{360}{c}\left(1+\frac{386}{5 c}+\ldots\right) .
\end{aligned}
$$

Obviously, the way we arrived at this proposal is rather cumbersome and one would expect that more streamlined derivations should be possible. We are convinced that the non-trivial cancellations that occured in our computations are a true sign of the integrability of this quantum field theory.

We remark that the result for $k_{c}$ is consistent with the formula

$$
k_{c}=-\frac{1}{48}(50-c+\sqrt{(c-2)(c-98)})-3 \text {, }
$$

which is the conjectured outcome of a KPZ type analysis of constraints in a more covariant formulation of $W_{3}$ gravity $[32,9]$. A proof of this formula will have to wait for a more rigorous formulation of the covariant theory. Recently, the following all-order results for the $Z$ factors have been conjectured [17]:

$$
Z^{(t)}=\frac{1}{2\left(k_{c}+3\right)}, \quad Z^{(w)}=\frac{\sqrt{30}}{\sqrt{\beta\left(k_{c}+3\right)^{3 / 2}}} .
$$

They correctly reproduce the singularity structure that one expects, and are in agreement with the expansions eq. (4.78).

The all-order result for the effective action, which is defined to be the Legendre transform of $W[t, w]$, follows from (4.77):

$$
\Gamma_{\text {eff }}[h, b]=2 k_{c} \Gamma_{L}\left[\frac{1}{2 k_{c} Z^{(t)}} h, \frac{30}{2 k_{c} Z^{(w)}} b\right] .
$$




\section{Concluding remarks}

The main new feature of $W$ gravity theories is that the underlying algebraic structure is a non-linearly generated Lie algebra. The fact that composite operators appear in the r.h.s. of commutators considerably complicates quantum $W$ gravity. Indeed, both the loop expansion due to integration over the matter fields and that due to the integration over the gauge fields induce subleading non-local terms in a $1 / c$ expansion of the quantum theory. In particular, there are non-local terms in the Ward identities for the induced action for finite $c$, which make it impossible (at least for us) to obtain the induced action in closed form. However, as we just saw for the case of $W_{3}$, the subleading non-localities precisely cancel each other when one computes the effective action, with as net result that the effective action for finite $c$ is, up to multiplicative renormalizations, given by the induced action in the large $c$ limit.

It would be interesting to see if the magical cancellations occuring in $W_{3}$ gravity can be understood from the point of view of an underlying simpler theory. For example, one might try to obtain this theory by reducing a 'master $W$ gravity' based on some version of $W_{\infty}$ or $W_{1+\infty}$. Among the candidates for this are the algebras $W_{\infty}(k)$ [38], which truncate to $W_{N}$ if $k=-N$ (note that these are again non-linear algebras). It is not yet clear to $n$ whether or not such reductions could indeed clarify the structure of quantum $W_{3}$ gravity.

One of the major open problems in the study of $W$ gravities remains the understanding of the underlying geometry. In the previous we saw that the propagating degrees of freedom of quantum $W$ gravity in the chiral gauge are generalizations of Beltrami differentials. A deeper insight into the meaning of these differentials may be obtained when one formulates $W$ gravity in a ' $W$ superspace'. Some preliminary results on the geometry were obtained in $[39,21,14,40,41]$.

Presently, we are investigating the covariant formulation of induced $W$ gravity [27]. This amounts to a covariant Lagrangian description of the work presented in [42]. Quite naturally, this results in a Yang-Mills like formulation of $W_{n}$ gravities, where the gauge group is the $W_{n}$ anti-de Sitter group: $S l(n)$. Starting from this one might hope, through the theory of cosets and induced representations, to gain a good understanding of $W$ geometry. This would be analogous to the construction of superspace supergravity theories along the lines of [43], combined with the construction of the geometry through homogenous spaces (a review of this can be found in [44]). 


\section{References}

[1] C.M. Hull, Phys. Lett. 240B (1990) 110

[2] K. Schoutens, A. Sevrin and P. van Nieuwenhuizen, Phys. Lett. 243B (1990) 245

[3] K. Schoutens, A. Sevrin and P. van Nieuwenhuizen, Nucl. Phys. B349 (1991) 791

[4] E. Bergshoeff, A. Bilal and K. S. Stelle, preprint CERN-TH.5924/90; C.M. Hull, Nucl. Phys. B353 (1991) 707

[5] C.M. Hull, contribution in this volume; K. Schoutens, A. Sevrin and P. van Nieuwenhuizen, contribution to the Festschrift on the occasion of M. Veltman's 60th birthday, preprint ITP-SB-91-34

[6] A.B. Zamolodchikov, Theor.Math.Phys. 63 (1985) 1205

[7] K. Schoutens, A. Sevrin and P. van Nieuwenhuizen, in Proceedings of the Trieste conference on Topological methods in field theory, Int.Jour.Mod.Phys. A6 (1991) 2891; Phys. Lett. 255B (1991) 549

[8] E. Bergshoeff, C.N. Pope, L.J. Romans, E. Sezgin, X. Shen and I.S. Stelle, Phys. Lett. 243B (1990) 350; K. Schoutens, A. Sevrin and P. van Nieurvenhuizen, Phys. Lett. 251B (1990) 355

[9] Y. Matsuo, Phys. Lett. 227B (1989) 209; in proceedings of the meeting Geometry and Physics, Lake Tahoe, July 1989

[10] K. Schoutens, A. Sevrin and P. van Nieuwenhuizen, Nucl. Phys. B364 (1901) 584

[11] C.M. Hull, Phys. Lett. 265B (1991) 347; preprint QMW/PH/91/2

[12] K. Schoutens, A. Sevrin and P. van Nieuwenhuizen, in Proceedings of the Jan. 1991 Miami Workshop on Quantum Field Theory, Statistical Mechanics, Quantum Groups and Topology, (Plenum, 1991)

[13] C.M. Hull, preprint QMW/PH/91/14; C.M. Hull and L. Palacios, preprint QNW/PH/91/15

[14] H. Ooguri, K. Schoutens. A. Sevrin and P. van Nieuwenhuizen, preprint ITPSB-91-16, RIMS-764 
[15] K. Schoutens, A. Sevrin and P. van Nieuwenhuizen, preprint ITP-SB-91-21, Nucl. Phys. B in print

[16] A. Ceresole, M. Frau, J. McCarthy and A. Lerda, Phys. Lett. 265B (1991) 72

[17] J. de Boer, private communication

[18] J. Thierry-Mieg, Phys. Lett. 197B (1987) 368

[19] K. Schoutens, A. Sevrin and P. van Nieuwenhuizen, Comm. Math. Phys. 124 (1989) 87

[20] C.N. Pope, L.J. Romans and K.S. Stelle, Phys. Lett. 268B (1991) 167; C.N. Pope, contribution in this volume

[21] S.R. Das, A. Dhar and K.S. Rama, preprints TIFR/TH/91-11, TIFR/TH/9120

[22] C.N. Pope, L.J. Romans and K.S. Stelle, Phys. Lett. 269B (1991) 287; C.N. Pope, L.J. Romans, E. Sezgin and K.S. Stelle, preprint CTP-TAMU-68/91

[23] E. Witten, Comm. Math. Phys. 92 (1984) 455

[24] A. M. Polyakov and P. B. Wiegmann, Phys. Lett. 131B (1983) 121; Phys. Lett. 141B (1984) 223.

[25] O. Alvarez, Nucl. Phys. B238 (1984) 61

[26] P. Di Vecchia and P. Rossi, Phys. Lett. 140B (1984) 344; P. Di Vecchia, B. Durhuus and J. L. Petersen, Phys. Lett. 144B (1984) 245.

[27] K. Schoutens, A. Sevrin and H. Verliride, in progress

[28] V.G. Knizhnik and A.B. Zamolodchikov, Nucl. Phys. B247 (1984) 83

[29] V.G. Knizhnik, A.M. Polyakov and A.B. Zamolodchikov, Mod.Phys.Lett. A3 (1988) 819

[30] A. M. Polyakov, Mod. Phys. Lett. A2 (1987) 893

[31] Al.B. Zamolodchikov, preprint ITEP 84-89 (1989)

[32] M. Bershadsky and H. Ooguri, Comm. Math. Phys. 126 (1989) 49

[33] A. Alekseev and S. Shatashvili, Nucl. Phys. B323 (1989) 719 
[34] L.J. Romans, Nucl. Phys. B352 (1991) 829

[35] A. M. Polyakov, Int. Jour. Mod. Phys. A5 (1990) 833

[36] A. Das, W.-J. Huang and S. Roy, preprint UR 1197, ER 13065-652

[37] A. Bilal, V.V. Fock and I.I. Kogan, Nucl. Phys. B359 (1991) 635

[38] I. Bakas and E. Kiritis, preprint UCB-PTH-91/44

[39] C.M. Hull, Phys. Lett. 269B (1991) 257

[40] G. Sotkov and M. Stanishkov, preprint ISAS-70/90/EP (June, 1990); G. Sotkov, M. Stanishkov and C.J. Zhu, preprint ISAS-75/90/EP (June 1990)

[41] J.-L. Gervais and Y. Matsuo, preprint LPTENS-91/29

[42] F.A. Bais, T. Tjin and P. van Driel, Nucl. Phys. B357 (1991) 632

[43] M. Kaku, P. K. Townsend and P. van Nieuwenhuizen, Phys. Rev. D17 (1978) 3179

[44] P. van Nieuwenhuizen, in Proceedings of Supersymmetry and Supergravity '84 (World Scientific, 1984) 

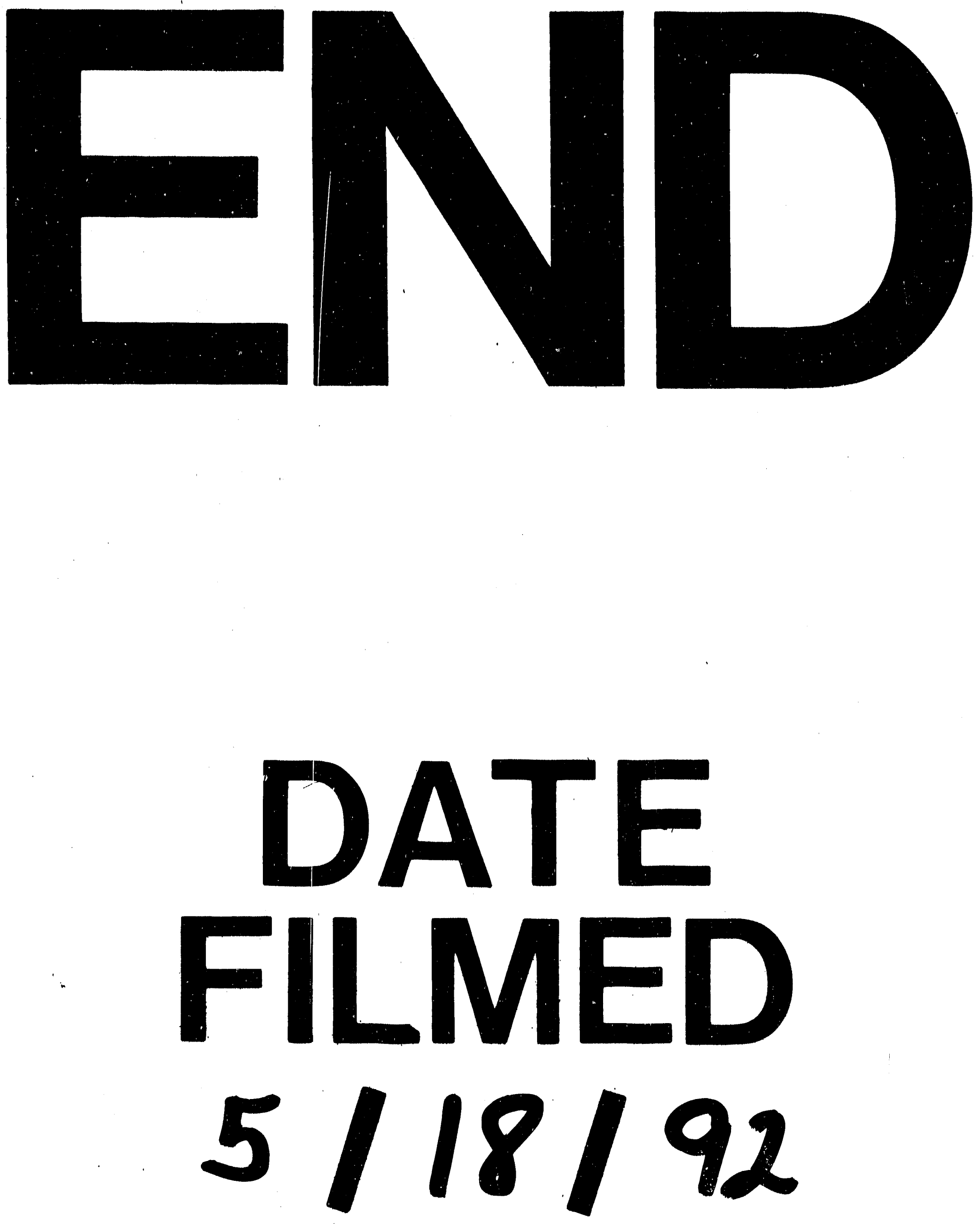
